BNL-112488-2016-JA

\title{
Two-Dimensional Superconductivity in the Cuprates Revealed by Atomic-Layer-by-Layer Molecular Beam Epitaxy
}

\author{
A. T. Bollinger and I. Božović \\ Submitted to Superconductor Science and Technology \\ July 2016 \\ Condensed Matter Physics and Material Science Department \\ Brookhaven National Laboratory
}

\section{U.S. Department of Energy USDOE Office of Science (SC), Basic Energy Sciences (BES) (SC-22)}

Notice: This manuscript has been authored by employees of Brookhaven Science Associates, LLC under Contract No. DE-SC0012704 with the U.S. Department of Energy. The publisher by accepting the manuscript for publication acknowledges that the United States Government retains a non-exclusive, paid-up, irrevocable, world-wide license to publish or reproduce the published form of this manuscript, or allow others to do so, for United States Government purposes. 


\section{DISCLAIMER}

This report was prepared as an account of work sponsored by an agency of the United States Government. Neither the United States Government nor any agency thereof, nor any of their employees, nor any of their contractors, subcontractors, or their employees, makes any warranty, express or implied, or assumes any legal liability or responsibility for the accuracy, completeness, or any third party's use or the results of such use of any information, apparatus, product, or process disclosed, or represents that its use would not infringe privately owned rights. Reference herein to any specific commercial product, process, or service by trade name, trademark, manufacturer, or otherwise, does not necessarily constitute or imply its endorsement, recommendation, or favoring by the United States Government or any agency thereof or its contractors or subcontractors. The views and opinions of authors expressed herein do not necessarily state or reflect those of the United States Government or any agency thereof. 


\title{
Two-Dimensional Superconductivity in the Cuprates Revealed by Atomic-Layer-by- Layer Molecular Beam Epitaxy
}

\author{
A. T. Bollinger ${ }^{1}$ and I. Božović ${ }^{1,2}$ \\ ${ }^{1}$ Condensed Matter Physics and Materials Science Division, Brookhaven National Laboratory, Upton NY 11973, \\ USA \\ ${ }^{2}$ Applied Physics Department, Yale University, New Haven CT 06520, USA
}

\begin{abstract}
Various electronic phases displayed by cuprates that exhibit high temperature superconductivity continue to attract much interest. We provide a short review of several experiments that we have performed aimed at investigating the superconducting state in these compounds. Measurements on single-phase films, bilayers, and superlattices all point to the conclusion that the high-temperature superconductivity in these materials is an essentially quasi-two dimensional phenomenon. With proper control over the film growth, high-temperature superconductivity can exist in a single copper oxide plane with the critical temperatures as high as that achieved in the bulk samples.
\end{abstract}

\section{Introduction}

High-temperature superconductivity (HTS) was discovered in the cuprates thirty years ago and remains one of the most important problems of condensed matter physics. Clearly many of the normal state properties are unusual, from the pseudogap phase ${ }^{1}$ as well as the various forms of charge and spin ordering ${ }^{2-10}$ on the underdoped side of the cuprate phase diagram, to the temperature-linear resistivity ${ }^{11-13}$ at higher doping levels and temperatures. The superconducting state in these materials is no less interesting, displaying unconventional pairing. The cuprates are complex oxides that form many phases, some of which are more stable than the targeted HTS compound so they are easily nucleated, forming unwanted secondary-phase precipitates. Hence, careful material synthesis must be performed in order to produce clean and homogeneous single-phase samples, which is necessary to investigate the unusual properties found in these compounds and differentiate the intrinsic properties from the extrinsic. We report here on several experiments performed on cuprate thin films grown using a custom-built molecular beam epitaxy system that allows for atomic-layer-by-layer synthesis with a high degree of control over film growth ${ }^{14-17}$. The results of this body of work unambiguously reveal another peculiar trait of the superconductivity in the cuprates that it is essentially quasi-two dimensional (2D) in nature.

Molecular beam epitaxy (MBE) is an ultra-high vacuum technique for the deposition of thin films. In our experiments, the film growth is performed very slowly (one monolayer per minute or less). The substrate temperature is kept within a tightly controlled window; we keep the temperature high enough to ensure considerable surface mobility, resulting in single crystal films that are atomically smooth and perfect, but low enough that bulk mobility and diffusion are minimized, allowing us to grow heterostructures and superlattices with atomically sharp interfaces. The film growth is monitored by reflection high-energy electron diffraction (RHEED) in real time to ensure the above. Oxygen is introduced into the films by flooding the area around the substrate with pure distilled ozone, the best oxygenating agent known, during the entire film growth. The molecular flux from each Knudsen cell on our system can be measured by atomic absorption spectroscopy in real time, with the rate data fed into the system control software that regulates how long the shutters on each source are open. The deposition process is controlled very accurately in this way, at the level of one percent of an atomic monolayer or better, allowing us to achieve digital, atomic-layer-by-layer (ALL) growth. The controlling software also allows the stoichiometry within each monolayer to be varied, providing flexibility in growing different compounds. In particular, the ALL-MBE technique is well suited, by design, for depositing alternating layers with great precision, and hence for the synthesis 
of artificial superlattices. The resulting films have rms surface roughness that can be as small as 1-2 $\AA$ as measured by atomic force microscopy. RHEED images also display an intense specular spot indicative of an atomically flat surface.

After the film growth is complete different annealing procedures are performed to further ensure the uniformity of the films, primarily targeting the oxygen content in the film as this is the element we have the least direct control over during growth. The details of the annealing depend on the material that was grown. Optimally doped and overdoped cuprate films are ozone annealed in situ, remaining at high temperature in an ozone atmosphere in the MBE chamber immediately after the film growth has completed. Moderate to heavily overdoped cuprate films are sometimes further annealed ex situ in oxygen using a high pressure furnace, as it is difficult to fully oxygenate these materials and the oxygen content may be slightly non-stoichiometric. Underdoped cuprate films, on the other hand, frequently take in too much oxygen and require lower temperature $\left(\sim 300{ }^{\circ} \mathrm{C}\right)$ vacuum annealing after growth to remove the excess oxygen that resides interstitially in the crystal lattice.

\section{Multilayer Structures}

\subsection{S-S'-S Superlattices}

The ability of ALL-MBE to deposit materials one atomic layer at a time is not only valuable when growing inherently layered materials such as the cuprates but also for fabrication of heterostructures consisting of multiple layers that combine different material properties. Several families of copper oxides display a variety of behaviors, depending on the doping level, i.e., as the carrier concentration in the material is adjusted either through cation substitution or by adding interstitial oxygen or removing it. For instance, $\mathrm{La}_{2} \mathrm{CuO}_{4}$ is an antiferromagnetic insulator that becomes superconducting when some strontium is substituted for lanthanum or when interstitial oxygen is introduced into the lattice by annealing. With further doping superconductivity completely disappears although the material becomes even more metallic. Therefore, one can stack layers of $\mathrm{La}_{2-\mathrm{x}} \mathrm{Sr}_{\mathrm{x}} \mathrm{CuO}_{4}$ (LSCO) doped to different levels, thus showing different electronic properties, in various arrangements. Such heterostructures built from members of the same material family gives the best chance for ideal interfaces since the lattice constants and electronic structures are almost identical.

The effective dimensionality of the HTS phenomenon was one of the first subjects studied by ALL-MBE that took advantage of the varying properties of different members within a single cuprate family. In this case the $\mathrm{Bi}_{2} \mathrm{Sr}_{2} \mathrm{Ca}_{n-1} \mathrm{Cu}_{n} \mathrm{O}_{2 n+4}$ system was used. ${ }^{18}$ The critical temperature for superconductivity, $T_{c}$, varies in these compounds with the number of copper oxide planes within a chemical formula unit. Single-phase films of the socalled 2201 compound $\left(\mathrm{Bi}_{2} \mathrm{Sr}_{2} \mathrm{CuO}_{6}\right)$ grown by ALL-MBE on $\mathrm{SrTiO}_{3}$ substrates showed $T_{c} \approx 15 \mathrm{~K}$. For the 2212 compound $\left(\mathrm{Bi}_{2} \mathrm{Sr}_{2} \mathrm{CaCu}_{2} \mathrm{O}_{8}\right)$ thin films were found to have a much higher $T_{c} \approx 85 \mathrm{~K}$. Superlattices containing these two materials were then grown with structural ordering that varied from one-half unit cell (UC) of 2201 for every $0.5 \mathrm{UC}$ of 2212 (1:1) up to $2.5 \mathrm{UC}$ of 2201 for every $0.5 \mathrm{UC}$ of 2212 (5:1). (One chemical formula corresponds to $0.5 \mathrm{UC}$ due to the presence of a crystallographic glide plane that doubles the unit cell).

These superlattices were nearly $1000 \AA$ thick, while still having atomically sharp interfaces as revealed by crosssectional transmission electron microscope images. ${ }^{19}$ Four-probe resistance measurements revealed that $T_{c}$ for all of the superlattices was the same as in the higher $T_{c} 2212$ compound (Fig. 1). This straightforward experiment showed that the separation between superconducting layers and their coupling had no effect on superconductivity and in that sense that HTS is $2 \mathrm{D}$ in nature.

\subsection{S-I-S Superlattices and Trilayers}

Similar results to those found with $\mathrm{Bi}_{2} \mathrm{Sr}_{2} \mathrm{Ca}_{n-1} \mathrm{Cu}_{n} \mathrm{O}_{2 n+4}$ have also been obtained in the LSCO material family. Several superlattices were grown by ALL-MBE containing layers of nearly optimally doped $\mathrm{La}_{1.85} \mathrm{Sr}_{0.15} \mathrm{CuO}_{4}$ that were one unit cell thick separated by thicker layers of insulating $\mathrm{La}_{2} \mathrm{CuO}_{4}$ (LCO). Susceptibility measurements (Fig. 2) on these superlattices showed that the 1 UC thick LSCO layers remained superconducting with a high $T_{c} \approx 30 \mathrm{~K}$ (the substrate was $\mathrm{SrTiO}_{3}$ ). ${ }^{20}$ There are only two copper oxide planes in one unit cell of LSCO so the fact that superconductivity occurred in these superlattices at all means that the copper oxide planes right next to the insulating LCO remained superconducting. Moreover, in these superlattices $T_{c}$ stayed close to those of single-phase films of $\mathrm{La}_{1.85} \mathrm{Sr}_{0.15} \mathrm{CuO}_{4}$ grown on the same type of substrate $\left(\mathrm{SrTiO}_{3}\right)$, implying that the carrier density in those copper-oxide planes was not much different either. 
The reverse geometry in which a 1 UC thick LCO layer was sandwiched between thicker layers of nearly optimally doped LSCO in the familiar $S-I-S$ trilayer arrangement were also explored. These trilayers were patterned into several mesa structures for each sample so that $c$-axis transport measurements could be performed. A superconducting transition due to the LSCO layers was observed that partially reduced the spreading resistance. Yet remarkably, no supercurrent was observed to pass from one side of these trilayers to the other, being completely blocked by the thin LCO layer, even at low bias current $(1 \mu \mathrm{A})$ and down to low temperature (4.2 K) (Fig. 3). The insulating LCO barriers were evidently free of pinholes and most interestingly were not affected by the superconducting layers that they were in such close vicinity to, i.e. no proximity effect was observed. Taken together, these experiments show that: (a) the HTS phase in optimally doped cuprates and the antiferromagnetic insulator phase do not mix, (b) that the interface between the two must be atomically sharp, and (c) that the HTS is confined to a region a single unit cell in thickness. ${ }^{20}$

\section{Interface Superconductivity}

\subsection{Bilayer Structures}

An even more striking example of quasi-2D superconductivity is found in heterostructures in which none of the constituent materials is superconducting in single-phase samples. A number of different bilayer films were grown consisting of insulating $\mathrm{La}_{2} \mathrm{CuO}_{4}$ (Fig. 4a) and metallic $\mathrm{La}_{1.55} \mathrm{Sr}_{0.45} \mathrm{CuO}_{4}$ (Fig. 4b) layers with various thicknesses. The resistance as a function of temperature, $R(T)$, for single phase films of the constituent compounds displays the expected insulating and metallic behavior. The bilayers (Fig. 4c), on the other hand, surprisingly show ${ }^{21}$ HTS with $T_{c} \approx 30 \mathrm{~K}$. The number of layers and their deposition sequence was found to have an influence on the observed $T_{c}$. Bilayers in which insulating layers were grown first and metallic layers second ("I-M bilayers") initially showed no superconductivity until about $2.5 \mathrm{UC}$ of metallic LSCO covered the insulating LCO. The critical temperature then rose sharply to $T_{c} \approx 15 \mathrm{~K}$ with $5 \mathrm{UC}$ or more of metallic LSCO coverage. This is in contrast to the inverse structure where metallic layers were grown first followed by insulating layers ("M-I bilayers"). Superconductivity appeared in these structures immediately when $0.5 \mathrm{UC}$ of LCO was deposited onto the metallic LSCO and reached its $30 \mathrm{~K}$ maximum with $1.5 \mathrm{UC}$ of LCO coverage. These preliminary results indicated that HTS is confined to an extremely thin layer near the interface, but further techniques were needed to really pin it down.

One such method, resonant soft x-ray scattering, can measure the profile of the mobile charge carriers within a film and distinguish them from the cations that are present. An ALL-MBE grown superlattice containing fifteen periods that were composed of $2 \mathrm{UC}$ of $\mathrm{La}_{1.64} \mathrm{Sr}_{0.36} \mathrm{CuO}_{4}$ and $1 \mathrm{UC}$ of LCO per period was studied by this technique. ${ }^{22}$ Again, although the individual layers are not superconducting by themselves, as in the case of the bilayer structures, the superlattice was nonetheless found to be superconducting, with $T_{c}=38 \mathrm{~K}$. The resonant soft x-ray scattering results showed that some small amount of Sr diffusion did occur in the superlattice but only over a length scale of at most 1 UC. This cation diffusion, however, was not enough to account for the observed interface superconductivity by itself. The mobile carrier density determined from the scattering was found to be significantly higher than what could be accounted for by the measured strontium profile, revealing charge redistribution across the interface. In the first copper oxide plane on the nominally insulating side of the interface, i.e. in LCO, a charge accumulation up to 0.18 holes per copper atom was measured, which is very close to that in optimally doped films, explaining the origin of HTS in this superlattice, and by extension in the bilayer structures as well. Charge transfer from the metallic layers into the insulating layers resulted in enough charge carriers being present in the nearby copper oxide planes that superconductivity could appear.

\section{$3.2 \delta$-Doping Tomography}

While the above experiments revealed the existence of interface superconductivity in a cuprate system and determined its root cause, the exact length scale over which the effect spread was not definitively known. This was clarified in a further set of measurements that leveraged on the precise stoichiometry control afforded by ALL-MBE and the empirically known ${ }^{23}$ fact that substitutional replacement of some of the copper with a small amount of zinc in the copper oxide planes of LSCO and oxygen doped LCO significantly suppresses $T_{c}$. The exact mechanism behind this is debated but it is largely believed to be the result of a pair-breaking process as the carrier density in the copper oxide planes, which is primarily determined by the lanthanum-strontium oxide layers, remains unaffected by this isovalent substitution. ${ }^{24}$ 
Several bilayers were grown that consisted of a bottom layer of $\mathrm{La}_{1.55} \mathrm{Sr}_{0.45} \mathrm{CuO}_{4}$ that was $3 \mathrm{UC}$ thick and a top layer of $\mathrm{La}_{2} \mathrm{CuO}_{4}$, also $3 \mathrm{UC}$ in thickness. ${ }^{24}$ For each individual bilayer, $3 \%$ of the copper in just one of the copper oxide planes was replaced with zinc. If conditions were right for superconductivity to occur in that plane then this zinc " $\delta$-doping" should have the effect of reducing the $T_{c}$. The location of this modified copper oxide plane was different for each of the bilayers that were synthesized. Resistance measurements unambiguously showed that the critical temperatures for all bilayers were very close to each other with $T_{c}=32 \mathrm{~K} \pm 4 \mathrm{~K}$ except for those bilayers in which the modified copper oxide plane was the second one from the interface on the insulating side (Fig. 5). For those bilayers the critical temperature was considerably lower, $T_{c}=19 \mathrm{~K} \pm 3 \mathrm{~K}$. This clearly demonstrated that the highest $T_{c}$ superconductivity was confined to that lone atomic plane, which makes it the thinnest known superconductor. This firmly established the fact that HTS can exist in a single $\mathrm{CuO}_{2}$ plane and is therefore $2 \mathrm{D}$ in nature.

\subsection{Bilayer Critical Temperature}

When comparing the results across the bilayer and superlattice work mentioned above a brief discussion about the value of $T_{c}$ is warranted. Each copper oxide plane in a given structure has $T_{c}$ determined solely by its own local carrier concentration. In the case of bilayers, the strontium doping in the metallic layers affects the superconductivity near the interface by providing a certain amount of carriers to spill over into the insulating side of the structure. This was examined in detail in an LSCO-LCO (M-I type) bilayer study involving over 800 different base layer doping levels. ${ }^{25}$ The strontium content in the LSCO base layers varied from heavily overdoped $(x=0.47)$ to optimal doping $(x=0.15)$ and yet the $T_{c}$ in the bilayers were essentially constant. ${ }^{25}$ This is exactly what one would expect if one assumed that the chemical potential of the carriers is doping independent up to optimal doping and then beyond this point decreased linearly with doping. ${ }^{25,26}$ When the base layer is heavily overdoped more carriers are transferred across the interface and the first $\mathrm{CuO}_{2}$ plane on the insulating side is overdoped as well, with near optimal doping found for the second $\mathrm{CuO}_{2}$ plane. As the base layer doping is decreased fewer carriers are transferred across the interface and the first $\mathrm{CuO}_{2}$ plane on the insulating side becomes the one that is near optimum doping and thus the one with the highest $T_{c}$. The next and further $\mathrm{CuO}_{2}$ layers in $\mathrm{LCO}$ remain essentially undoped, because there is no chemical potential difference to drive further hole depletion and accumulation. This model explains the appearance of superconductivity in different locations for the LSCO-LCO bilayers and superlattices and also why no $T_{c}$ reduction was observed in the case of the S-I-S superlattices and trilayers. Note, however, that this 'broken' chemical potential dependence of doping is certainly not expected within the standard Fermi Liquid description, indicating an anomalous nature of the 'normal' state from which HTS develops.

Another parameter that has an effect on the critical temperature of the interface superconductivity in MBE grown bilayers is epitaxial strain, equivalent to its well known influence on $T_{c}$ in epitaxial single phase films. This is most clearly seen in the differences in $T_{c}$ for I-M and M-I bilayers, shown in Fig. 4c, which is $\sim 15 \mathrm{~K}$ and $\sim 30 \mathrm{~K}$, respectively. X-ray diffraction patterns ( $\omega-2 \theta$ scans) on these bilayers surprisingly show that they display a single Bragg peak, rather than two from each I and M section separately, even though the $c$-axis lattice parameters are fairly different for these two constituent materials. ${ }^{27}$ The peak position reveals that the top layer always adapts to the bottom one, i.e. the peak position for I-M bilayers corresponds with that of single phase $\mathrm{La}_{2} \mathrm{CuO}_{4}$ films while for M-I bilayers it matches that of single phase $\mathrm{La}_{1.55} \mathrm{Sr}_{0.45} \mathrm{CuO}_{4}$ films. Therefore the strain conditions where the highest $T_{c}$ superconductivity resides (the second copper oxide plane in the nominally insulating side from the interface in this case) is different for these two types of bilayers resulting in different critical temperatures. The impact that strain has on $T_{c}$ is evidently substantial and must be considered in addition the doping level in the metallic layer as discussed above.

\section{Field-Effect Controlled Superconductivity}

The 2D nature of the superconductivity found in the cuprates was revealed by another set of measurements that introduced carriers into the system by electrostatic means in a type of field-effect transistor (FET) geometry. Manipulating the carriers in a cuprate structure in this way can allow for fine carrier concentration tunability, whereas when relying on differences in chemical potentials between the different layers within a given structure to move carriers around the amount of transferred charge is permanently set by the stoichiometry of the constituent layers. However, achieving significant changes in the carrier density by field effect in cuprates is not as easy as in typical semiconductors since the intrinsic carrier density in superconducting cuprates is relatively high $\left(\sim 10^{21} \mathrm{~cm}^{-3}\right)$. The screening length in LSCO, for example, in about $0.5 \mathrm{UC}$, so only the topmost $\mathrm{CuO}_{2}$ plane is considerably affected. As a result, thin films of very high quality, such as those provided by the ALL-MBE technique, are required. The surface needs to be atomically smooth; the rms roughness must be much smaller than $0.5 \mathrm{UC}$, so that 
the topmost $\mathrm{CuO}_{2}$ layer is sufficiently connected. The requisite electric fields are also quite large at $10^{8} \mathrm{~V} / \mathrm{cm}$. While conventional top, back, and even ferroelectric gating schemes have been tried in the past ${ }^{28}$ electrolyte gating is a more effective method for the cuprates ${ }^{29}$ and is currently favored in basic research to induce the largest changes in carrier concentration. ${ }^{30,31}$

Several thin films of LSCO were grown by ALL-MBE that were 1-2 UC thick, with different doping levels that fell in the range from $x=0.06$ to $x=0.20$. The ionic liquid DEME-TFSI was used as a gate electrolyte between the sample and a gate electrode. When a voltage was applied to the gate electrode a Helmholtz double layer formed at the sample surface generating large shifts in the carrier concentration at the surface as well as in $T_{c}$ (up to $75-80 \%$ ) and in the normal-state resistance (Fig. 6a). ${ }^{32}$ These changes were reversible and reproducible. Shifts in $T_{c}$ were also observed by mutual inductance measurements using $\mathrm{NaF}$ dissolved in polyethylene oxide as the electrolyte. ${ }^{32}$

The most dramatic effects in this study were observed in underdoped films (grown on a non-conducting buffer layer), which by applying the electric field could be tuned from insulating behavior to superconducting with $T_{c}$ as high as $29 \mathrm{~K}$. The separatrix between superconducting and insulating states was found to be $R_{\square}=R_{Q}=h / 4 e^{2}$, the quantum resistance for pairs (Fig. 6a inset). The $R_{\square}(T)$ data for each field induced doping level were inverted, generating $R_{\square}(x)$ curves that all crossed at a critical value, $x \approx 0.06 \equiv x_{c}$, in good agreement with the known phase diagram of LSCO. Scaling analysis of the data showed that all measured curves collapsed onto a single curve (Fig. 6b), with critical exponent product $z v \approx 3 / 2$. These findings are consistent with a 2D superconductor-insulator quantum phase transition that is bosonic in nature, i.e. the transition is driven by superconducting phase fluctuations and both pairs and vortices exist on both sides of the transition. Cooper pairs are mobile and vortices bound in vortex-antivortex pairs in the superconducting phase, whereas vortices are unbound and mobile with Cooper pairs localized in the insulating phase. Very similar results were achieved in $\mathrm{YBa}_{2} \mathrm{Cu}_{3} \mathrm{O}_{7-\delta}$ thin films, indicating that this behavior is universal to cuprate superconductivity. ${ }^{33}$

This work was supported by the U.S. Department of Energy, Basic Energy Sciences, Materials Sciences and Engineering Division.

\section{References}

1. T. Timusk and B. Statt, Rep. Prog. Phys. 62, 61 (1999)

2. W. D. Wise, M. C. Boyer, K. Chatterjee, T. Kondo, T. Takeuchi, H. Ikuta, Y. Wang, and E. W. Hudson, Nature Phys. 4, 696 (2008)

3. C. V. Parker, P. Aynajian, E. H. da Silva Neto, A. Pushp, S. Ono, J. Wen, Z. Xu, G. Gu, and A. Yazdani, Nature 468, 677 (2010)

4. T. Wu, H. Mayaffre, S. Krämer, M. Horvatić, C. Berthier, W. N. Hardy, R. Liang, D. A. Bonn, and M.-H. Julien, Nature 477, 191 (2011)

5. G. Ghiringhelli, M. Le Tacon, M. Minola, S. Blanco-Canosa, C. Mazzoli, N. B. Brookes, G. M. De Luca, A. Frano, D. G. Hawthorn, F. He, T. Loew, M. Moretti Sala, D. C. Peets, M. Salluzzo, E. Schierle, R. Sutarto, G. A. Sawatzky, E. Weschke, B. Keimer, and L. Braicovich, Science 337, 821 (2012)

6. J. Chang, E. Blackburn, A. T. Holmes, N. B. Christensen, J. Larsen, J. Mesot, R. Liang, D. A. Bonn, W. N. Hardy, A. Watenphul, M. v. Zimmerman, E. M. Forgan, and S. M. Hayden, Nature Phys. 8, 871 (2012)

7. D. H. Torchinsky, F. Mahmood, A. T. Bollinger, I. Božović, and N. Gedik, Nature Mater. 12, 387 (2013)

8. M. Le Tacon, A. Bosak, S. M. Souliou, G. Dellea, T. Loew, R. Heid, K-P. Bohnen, G. Ghiringhelli, M. Krisch and B. Keimer, Nature Phys. 10, 52 (2014)

9. E. H. da Silva Neto, P. Aynajian, A. Frano, R. Comin, E. Schierle, E. Weschke, A. Gyenis, J. Wen, J. Schneeloch, Z. Xu, S. Ono, G. Gu, M. Le Tacon, A. Yazdani, Science 343, 394 (2014)

10. J. Wu, A. T. Bollinger, Y. Sun, and I. Bozovic, Proc. Natl. Acad. Sci. USA 113, 4284 (2016)

11. J. Cava, B. Batlogg, R. B. von Dover, and E. A. Rietman, Phys. Rev. Lett. 58, 408 (1987)

12. J. Cava, B. Batlogg, R. B. von Dover, D. W. Murphey, S. Sunshine, T. Siegrist, J. P. Remeika, E. A. Rietman, S. Zahurak, and G. P. Espinosa, Phys. Rev. Lett. 58, 1676 (1987)

13. M. Gurvitch and A. T. Fiory, Phys. Rev. Lett. 59, 1337 (1987)

14. J. N. Eckstein, I. Bozovic, and G. F. Virshup, MRS Bull 19(9), 44 (1994) 
15. I. Bozovic, J. N. Eckstein, and G. F. Virshup, Physica C 235-240, 178 (1994)

16. J. N. Eckstein and I. Bozovic, Annual Reviews of Materials Science 25, 679 (1995)

17. I. Bozovic, IEEE Trans. Appl. Superconduct. 11, 2686 (2001)

18. I. Bozovic, J. N. Eckstein, M. E. Klausmeier-Brown, G. Virshup, Journal of Superconductivity 5, 19 (1992)

19. I. Bozovic, J. N. Eckstein, G. F. Virshup, A. Chaiken, M. Wall, R. Howell, M. Fluss, Journal of Superconductivity 7, 187 (1994)

20. I. Bozovic, G. Logvenov, M. A. J. Verhoeven, P. Caputo, E. Goldobin, and T. H. Geballe, Nature 422, 873 (2003)

21. A. Gozar, G. Logvenov, L. Fitting Kourkoutis, A. T. Bollinger, L. A. Giannuzzi, D. A. Muller, and I. Bozovic, Nature 455, 782 (2008)

22. S. Smadici, J. C. T. Lee, S. Wang, P. Abbamonte, G. Logvenov, A. Gozar, C. Deville Cavellin, and I. Bozovic, Phys Rev Lett 102, 107004 (2009)

23. G. Xiao, F. H. Streitz, A. Gavrin, Y. W. Du, and C. L. Chien, Phys. Rev. B 35, 8782(R) (1987)

24. G. Logvenov, A. Gozar, and I. Bozovic, Science 326, 699 (2009)

25. J. Wu, O. Pelleg, G. Logvenov, A. T. Bollinger, Y-J. Sun, G. S. Boebinger, M. Vanević, Z. Radović, and I. Božović, Nature Materials 12, 877 (2013)

26. A. Ino, T. Mizokawa, A. Fujimori, K. Tamasaku, H. Eisaki, S. Uchida, T. Kimura, T. Sasagawa, and K. Kishio, Phys Rev Lett 79, 2101 (1997)

27. V. Y. Butko, G. Logvenov, N. Božović, Z. Radović, and I. Božović, Adv. Mater. 21, 3644 (2009)

28. C. H. Ahn, A. Bhattacharya, M. Di Ventra, J. N. Eckstein, C. Daniel Frisbie, M. E. Gershenson, A. M. Goldman, I. H. Inoue, J. Mannhart, A. J. Millis, A. F. Morpurgo, D. Natelson, and J.-M. Triscone, Rev. Mod. Phys. 78, 1185 (2006)

29. S. G. Haupt, D. R. Riley, C. T. Jones, J. Zhao, and J. T. McDevitt, J. Am. Chem. Soc. 115, 1196 (1993)

30. K. Ueno, S. Nakamura, H. Shimotani, A. Ohtomo, N. Kimura, T. Nojima, H. Aoki, Y. Iwasa and M. Kawasaki, Nature Mater. 7, 855 (2008)

31. J. T. Ye, S. Inoue, K. Kobayashi, Y. Kasahara, H. T. Yuan, H. Shimotani, and Y. Iwasa, Nature Mater. 9, 125 (2010)

32. A. T. Bollinger, G. Dubuis, J. Yoon, D. Pavuna, J. Misewich, and I. Božović, Nature 472, 458 (2011)

33. X. Leng, J. Garcia-Barriocanal, S. Bose, Y. Lee, and A. M. Goldman, Phys. Rev. Lett. 107, 027001 (2011) 


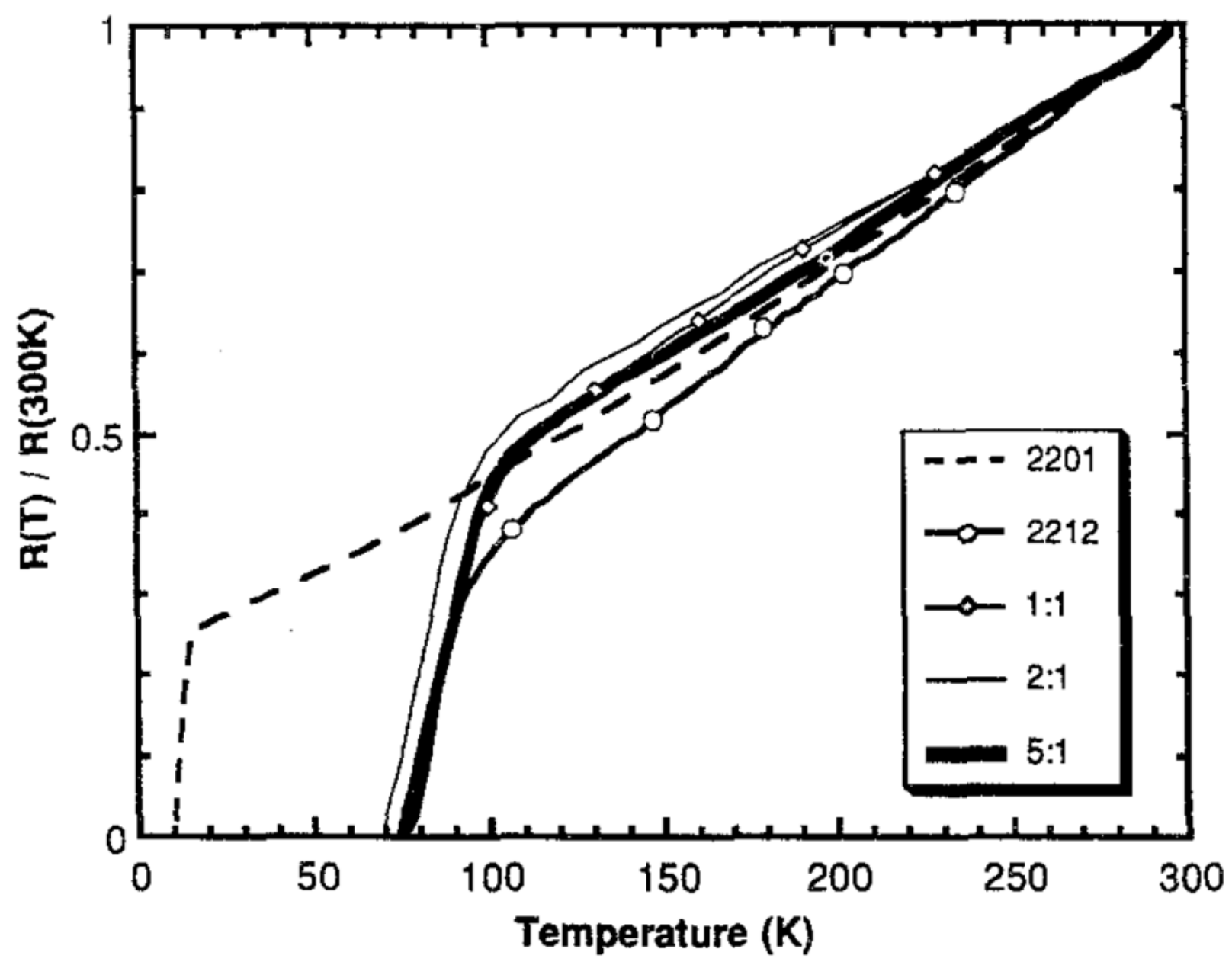

Figure 1. Resistive transitions of $\mathrm{MBE}$ grown $\mathrm{Bi}_{2} \mathrm{Sr}_{2} \mathrm{Ca}_{n-1} \mathrm{Cu}_{n} \mathrm{O}_{2 n+4}$ single-phase films and superlattices. All films and superlattices are $1,000 \AA$ thick. Single phase films of $\mathrm{Bi}_{2} \mathrm{Sr}_{2} \mathrm{CuO}_{6}$ and $\mathrm{Bi}_{2} \mathrm{Sr}_{2} \mathrm{CaCu}_{2} \mathrm{O}_{8}$ compounds are denoted as 2201 and 2212, respectively, while 1:1, 2:1, and 5:1 are $(1 \times 2201):(1 \times 2212),(2 \times 2201):(1 \times 2212)$, $(5 \times 2201):(1 \times 2212)$ superlattices, respectively. The fact that $T_{c}$ does not decrease when single 2212 layers are separated by as much as $60 \AA$ indicates that HTS in 2212 is $2 \mathrm{D}$ in nature. Reproduced with permission from Ref. 18. 


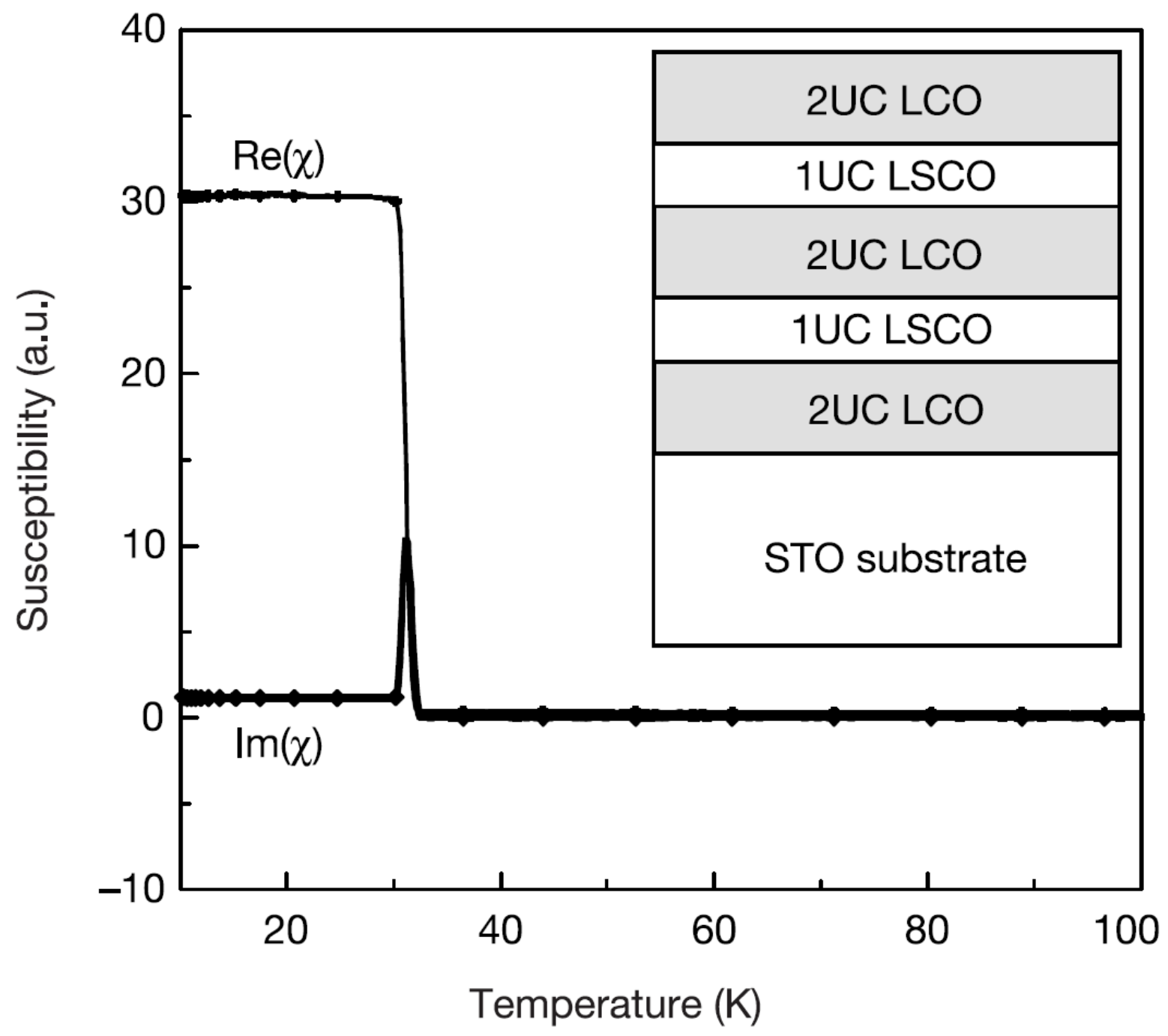

Figure 2. Susceptibility measurement of an I-S-I-S-I superlattice of LCO and optimally doped LSCO shows a very sharp superconducting transition and no indication of $T_{c}$ reduction. (Inset) Diagram of the superlattice layering sequence and layer thicknesses. Like in the 2201:2212 superlattices, independence of Tc on the separation of HTS layers implies that the phenomenon is 2D in nature. Reproduced with permission from Ref. 20. 


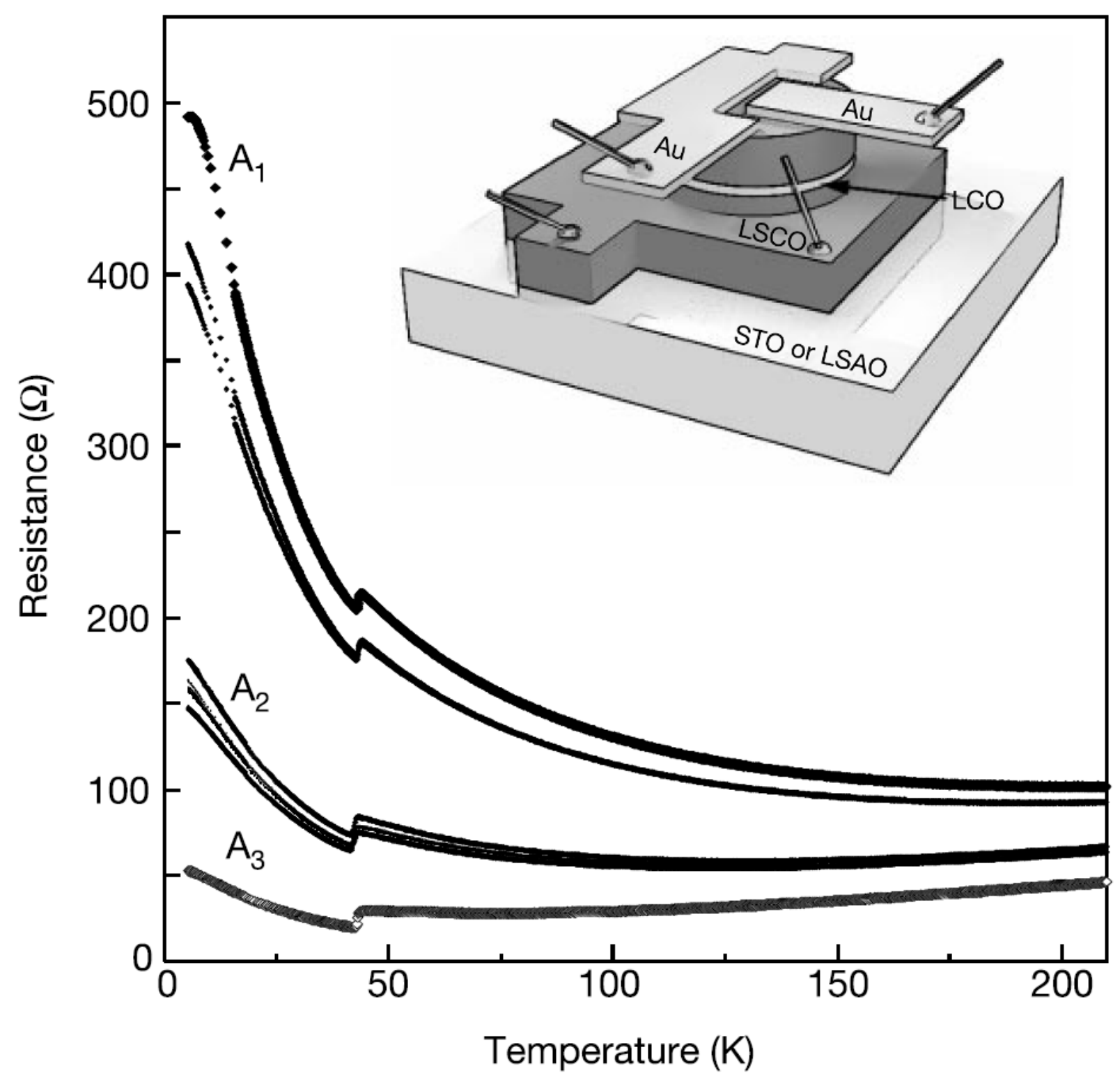

Figure 3. Resistance along the $c$-axis for several representative $S-I-S$ junctions. The nominal cross sectional area of the mesas is different for each grouping of curves $\left(A_{1}=180 \mu \mathrm{m}^{2}, A_{2}=310 \mu \mathrm{m}^{2}, A_{3}=710 \mu \mathrm{m}^{2}\right)$. When the resistance is scaled to the area, the curves essentially collapse to one another. The drop in resistance seen in each curve around $40 \mathrm{~K}$ is due to the superconducting transitions in the optimally doped LSCO layers. Below this temperature the resistance is solely from the LCO layer. (Inset) Illustration of the $S-I-S$ device layout. Reproduced with permission from Ref. 20. 

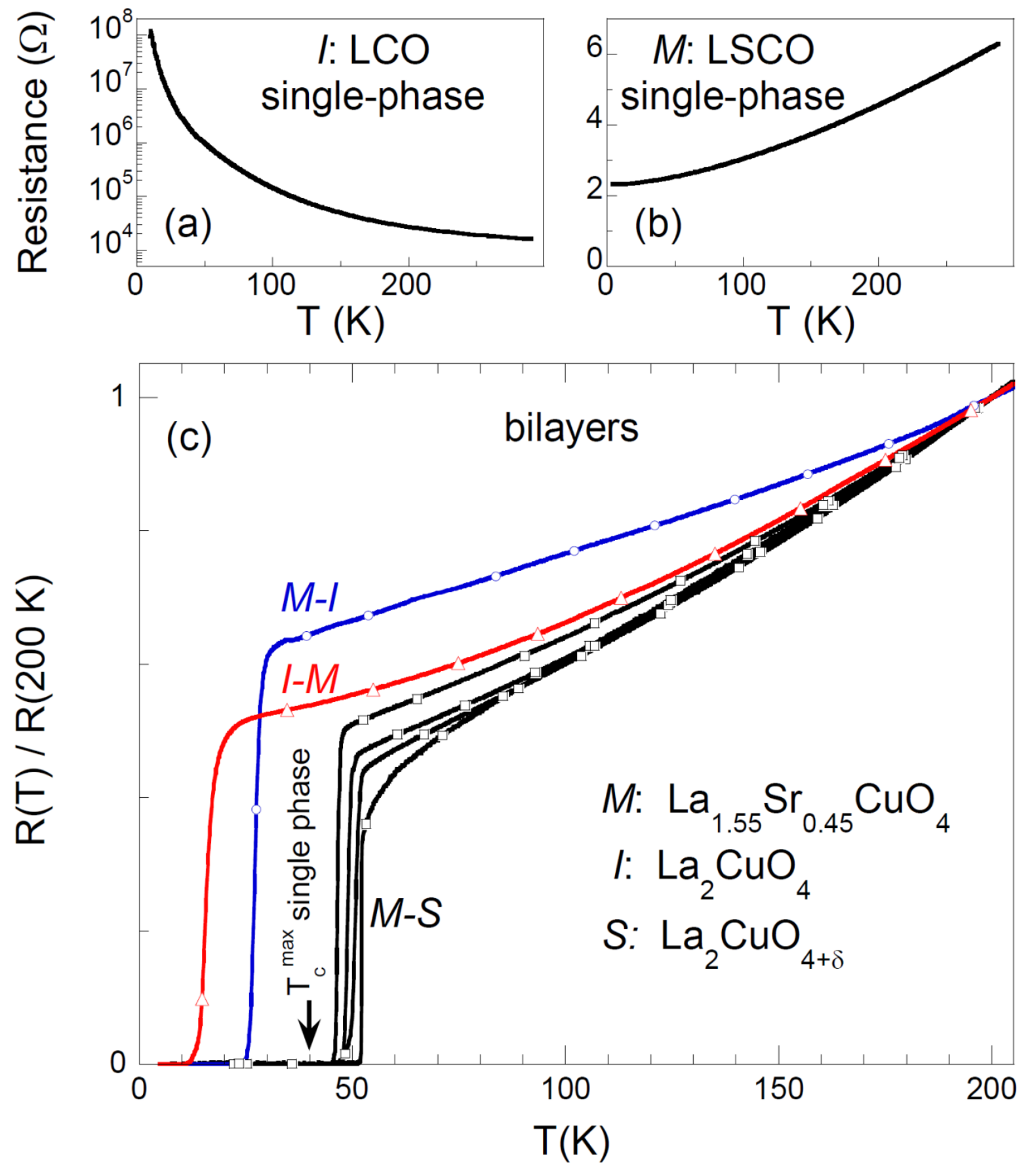

Figure 4. (a) Resistance as a function of temperature for a single phase $\mathrm{LaCuO}_{4}$ film showing the insulating behavior $(I)$ expected from undoped cuprate compounds. (b) The same, for a single phase $\mathrm{La}_{1.55} \mathrm{Sr}_{0.45} \mathrm{CuO}_{4}$ film, showing metallic behavior $(M)$ without superconductivity, as expected from heavily overdoped cuprate compounds. (c) When $M$ and $I$ are grown on top of one another, however, superconducting behavior is observed with $T_{c}$ that depends on the layering sequence. Furthermore, when bilayers with $\mathrm{LaCuO}_{4}$ layers on top are annealed in ozone all of the LCO layers become superconducting as expected due to the excess oxygen, but $T_{c}$ becomes unexpectedly high, $25 \%$ higher than in any single-phase LSCO film grown under similar conditions. Reproduced with permission from Ref. 21. 

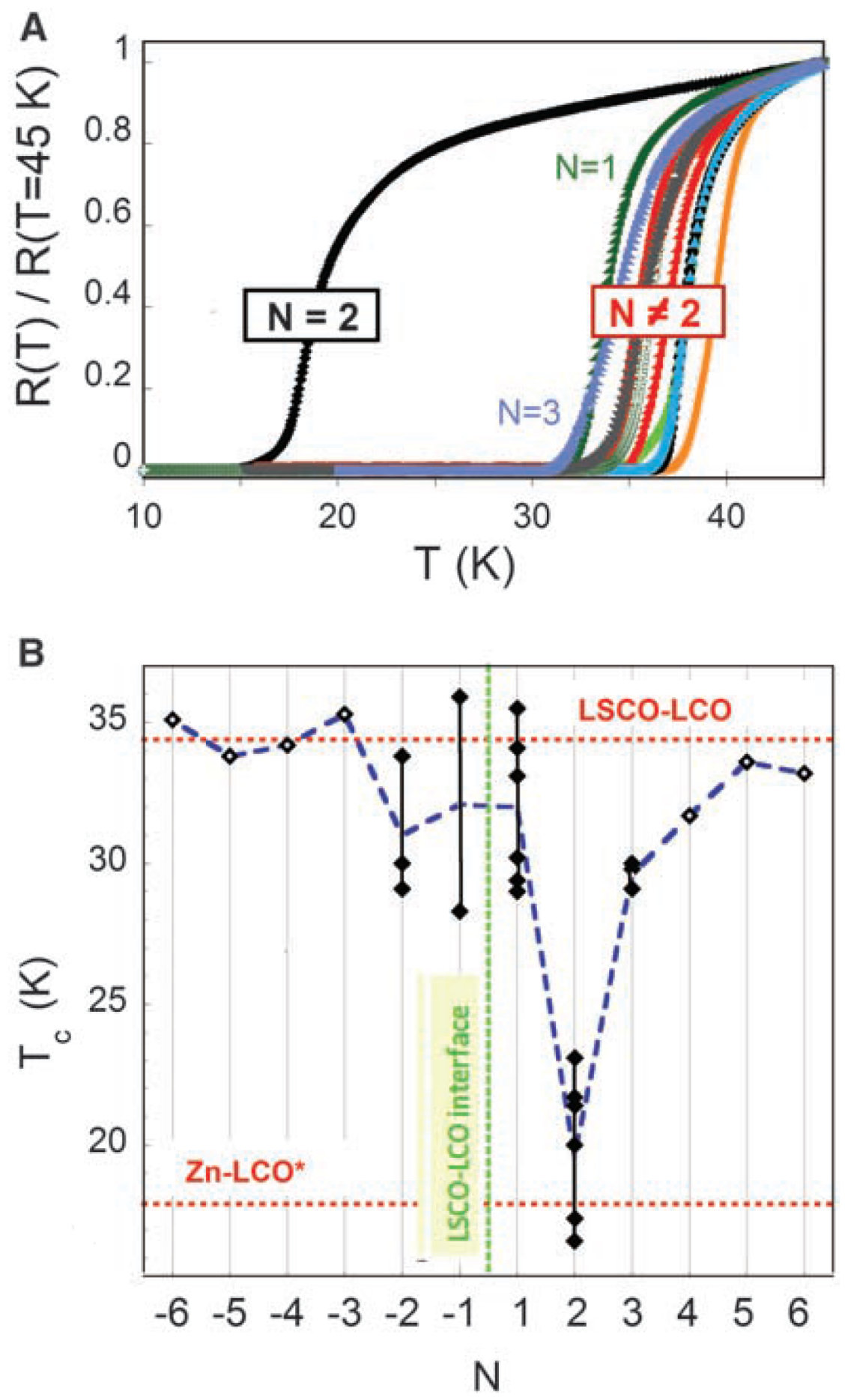

Figure 5. (a) Resistance data for several $\left(6 \times \mathrm{La}_{1.55} \mathrm{Sr}_{0.45} \mathrm{CuO}_{4}\right) /\left(6 \times \mathrm{LaCuO}_{4}\right)$ bilayer films in which $3 \%$ of the copper in a single $\mathrm{CuO}_{2}$ plane is replaced with zinc. The location of this $\delta$-doped plane, identified by the index $N$, is different for each bilayer shown. The critical temperatures for all of the bilayers is the same (within some sample to sample variation) except for one bilayer. The zinc doping in this bilayer occurred in the second $\mathrm{CuO}_{2}$ plane from the interface in the $\mathrm{LaCuO}_{4}$ side. (b) $T_{c}$ data for a number of bilayers confirm that $\mathrm{Zn} \delta$-doping this particular plane (and none of the others) significantly reduces the critical temperature of the bilayer structure, demonstrating that the highest $T_{c}$ superconductivity is confined to a single $\mathrm{CuO}_{2}$ plane. Reproduced with permission from Ref. 24 . 

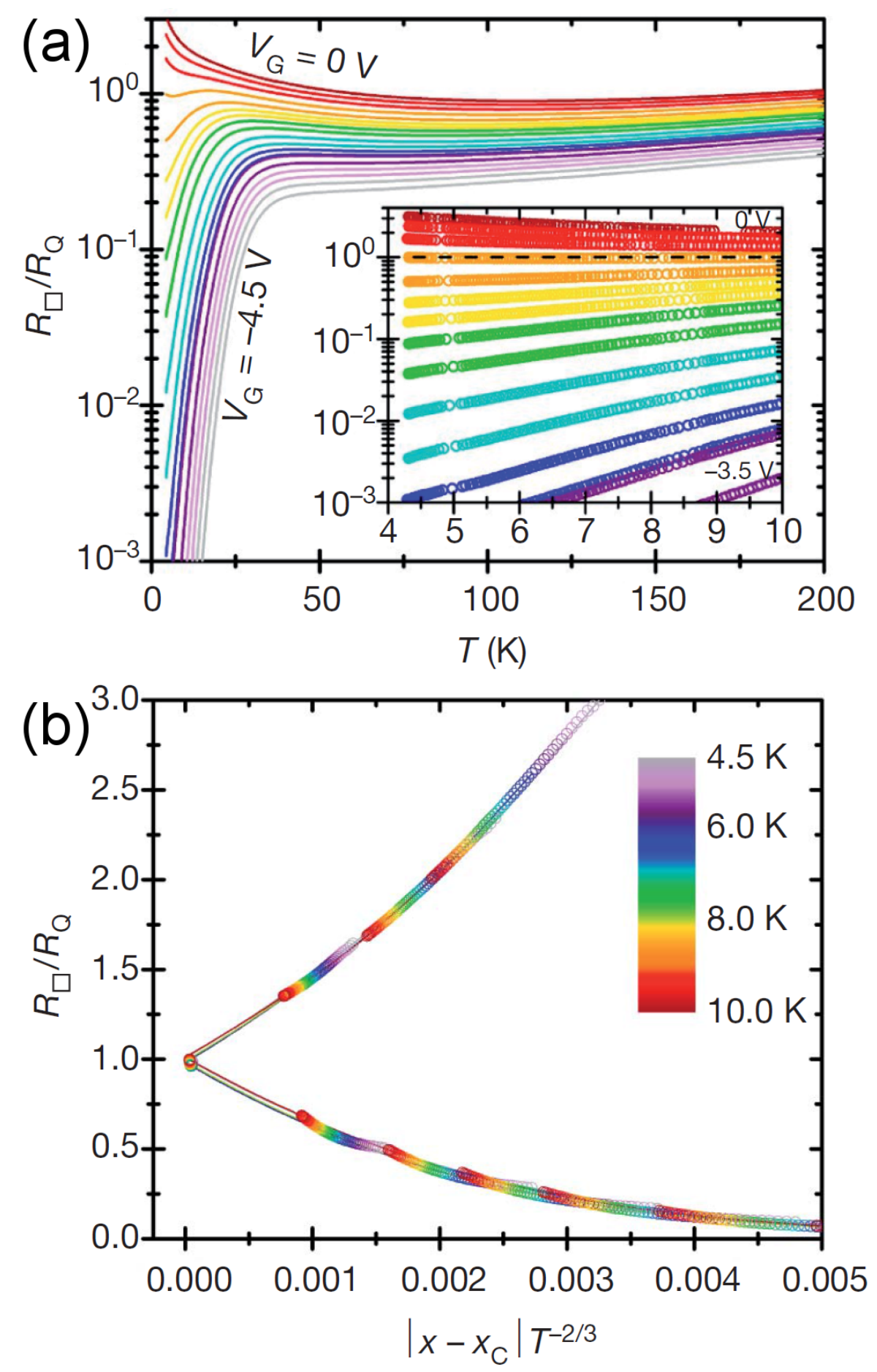

Figure 6. (a) A thin film of underdoped LSCO, initially displaying insulating behavior, becomes more conductive and eventually superconducting under the influence of an increasingly greater electric field generated by applying a gate voltage across an electrolyte (a polymer or an ionic liquid). The superconductor-insulator transition (SIT) in this film occurred at the sheet resistance $R_{\square}=R_{Q}=h /(2 e)^{2}=6.45 \mathrm{k} \Omega$, suggestive of electron pairing on both sides of the SIT. The effective doping level for this sample is varied from $x=0.051$ for $V_{G}=0 \mathrm{~V}$ to $x=0.139$ for $V_{G}=-4.5$ V. (b) Scaling of the $R_{\square}(T)$ curves, as expected for a quantum critical behavior near SIT in a $2 \mathrm{D}$ system. Reproduced with permission from Ref. 32. 